\title{
Comparison the Muscle Activation in the Trunk and Lower Limbs of Subjects Wearing High-Heeled or Flat Shoes While Crossing Over Obstacles of Different Heights*
}

\author{
Jin-Seong Park $\cdot$ Jin-Tae $\operatorname{Han}^{1 \dagger}$ \\ Daonwellness Corporation \\ ${ }^{1}$ Dept. of Physical Therapy, Kyungsung University
}

Received: July 18, 2017 / Revised: July 19, 2017 / Accepted: July 26, 2017

(c) 2017 J Korean Soc Phys Med

\begin{abstract}
| Abstract |
PURPOSE: The purpose of this study was to compare muscle activation of the trunk and lower limbs of subjects wearing high-heeled or flat shoes while crossing over obstacles of different heights.
\end{abstract}

METHODS: Twenty subjects participated in this study. While wearing high-heeled shoes $(7 \mathrm{~cm})$ or flat shoes $(0 \mathrm{~cm})$, the subjects were asked to cross over obstacles of different heights $(10 \%, 20 \%$, and $30 \%$ of their lower-limb length). Muscle activation of the trunk and lower limbs with the supported side while crossing over obstacles of different heights was measured using the electromyogram (Noraxon, DTS, Germany). Two-way repeated ANOVA was used to compare the muscle activation between high-heel shoes and flat shoes while crossing over obstacles of different heights. All statistical analyses were performed using SPSS ver. 21,

\footnotetext{
* This paper is abridgment of thesis for a master's degree of Jin-Seong Park (2015).

† Corresponding Author : jthan2001@ks.ac.kr

This is an Open Access article distributed under the terms of the Creative Commons Attribution Non-Commercial License (http://creativecommons.org/licenses/by-nc/3.0) which permits unrestricted non-commercial use, distribution, and reproduction in any medium, provided the original work is properly cited.
}

and p-values less than .05 were used to identify significant differences.

RESULTS: As an obstacle's height increased, muscle activation of the trunk and lower limbs with the supported side was increased while wearing either type of shoe, and it was generally higher while wearing high-heeled shoes. However, tibialis anterior muscle activity while wearing high-heeled shoes was lower than while wearing flat shoes.

CONCLUSION: This study showed that muscle activation of the trunk and lower limbs was higher when subjects wore high-heeled shoes than when they wore flat shoes while crossing over obstacles of different heights. Therefore, high-heeled shoes can easily cause high muscle fatigue of the trunk and lower limbs, and the TA muscle may weaken in persons who wear high-heeled shoes.

Key Words: High-heeled shoes, Muscle activity, Obstacle

\section{Introduction}

Shoes facilitate the natural movements of the lower limb that occur during gait to supplement the functionality of the feet while absorbing the impact from the ground to protect the feet, and shoes play a significant role in prevent- 
ing injuries. Shoes were developed with the goal of preventing injuries and reducing the loads imposed on the human body; shoes have been used diversely depending on the form of exercise, speed, posture, number of repetitions, ground condition, and personal characteristics. However, in the past, the roles of shoes and their benefits have rarely been considered, and changes in muscle activity related to balance and gaits due to shoe wearing have not been considered important (Menz and Lord, 1999). According to many studies, high-heel shoes elevate the medial longitudinal arch of the sole. Feet with heightened medial longitudinal arches are associated with functional problems and damage to the musculoskeletal system (Cowan et al., 1994). Additionally, high-heel shoes cause the body weight to be loaded mostly on the front part of the feet during gait (Blanchette et al., 2011), and heel height greatly affects the mobility and stability of the feet that to support natural gait and the maintenance of appropriate balance, shoes should have large areas that contact the ground, they should have low heels, and they should be constructed of materials that are soft and flexible (Powell et al., 2000). High-heel shoes may adversely affect gait and balance functions, such as increasing plantar flexion and changing body arrangements, and such effects prominently appear in the ankle joints (Snow et al., 1992, Lee et al., 2009). Such effects may also affect the activities of the muscles around the ankle joints. As heel heights increase, the calf muscle is shortened, and the tibialis anterior is extended, which leads to decreases in the contractile force of the tibialis anterior according to the relationship between the length and tension of the muscle. Therefore, wearing high-heel shoes induces weakness in the tibialis anterior and weakens the muscle strength of the lower leg while inducing dynamic changes in the feet and cause imbalance in the lower limb alignment and thereby limit the range of movement of the ankle joint (Abbud et al., 2009). Weakened lower limb muscle strength leads to reductions in gait speed, balance ability, and stair-climbing ability. Diverse changes in the musculoskeletal system, such as an ankle muscle strength reduc- tions, damage to ligaments, ankyloses, and inappropriate body arrangements, are causes of ankle instability (Garn and Newton, 1998). Wearing high-heel shoes increases the plantar flexion of the ankle joints, which changes the relative positions of the bones in the joints and the points of origin of the muscles. Eventually, wearing high-heel shoes leads to changes in the positions of body segments and the center of gravity, which result in kinetic changes to compensate for these other changes (Snow and Williams, 1994). Although the high-heel shoes preferred by most women are frequently mentioned as a risk factor that can cause problems not only in the feet and ankles but also in the entire body, studies of the changes in muscle activity that may appear due to high-heel shoe wearing and related physical changes are rare (Gefen et al., 2002). Additionally, few studies have been conducted on the effects of external environment factors, such as ground conditions and obstacles, on gait.

Obstacle gaits are movements that require highly harmonized joint movements in which the stance phase of the lower limbs should include accurate contact with the ground to maintain body balance, while the swing phase of the lower limb should involve fine adjusting movements (Chesnin et al., 2000). Obstacle gaits are complicated movement tasks that are related to many movements in daily living activities (Said et al., 2001). Despite the fact that gaits that are affected by external environments frequently occur during daily living activities, studies of this subject are very rare. The diagnosis and evaluation of gait in special situations in daily living, such as negotiating environmental obstacles, are important for patients (Power et al., 1997; Han, 2014).

In this respect, the present study was conducted to measure and compare changes in the activities of stance-side trunk muscles and lower limb muscles while subjects wearing high-heel shoes walked over obstacles. The study was designed to compare the activities of the stance-side trunk and lower limb muscles while healthy adult females wearing different types of shoes (high-heel shoes and flat shoes) moved over obstacles that were $10 \%$, 
$20 \%$, or $30 \%$ of the height of their lower limbs. The present study was approved by the Institutional Review Board (IRB) of Kyungsung University.

\section{Methods}

\section{Subjects}

The present study was conducted with 20 young women who had been wearing high-heel shoes in their daily lives for at least four hours per day, three days per week, for at least one year (Snow and Williams, 1994). Those with neurologic or orthopedic ankle problems, any ankle injury within the previous six months, current discomfort or pain anywhere in the body, current pregnancy, or any psychiatric problem was excluded from the study. The subjects were instructed to kick a ball, and the foot they used to kick the ball was identified as the dominant foot (Edwards et al., 2008). The dominant foot of each subject was the left foot. The general characteristics of the study subjects were as follows: age, $22.18 \pm 1.57$ years old; height, $159.81 \pm 4.96$ $\mathrm{cm}$; and weight, $49.81 \pm 3.32 \mathrm{~kg}$.

\section{Apparatus}

Electromyography (Noraxon, DTS, Germany) was used as a measurement tool. The rectus abdominalis (RA), erector spinalis (ES), external oblique abdominalis (EO), and internal oblique abdominalis (IO) of the trunk were taken as the measurement regions, and the rectus femoris (RF), semitendinosus (ST), tibialis anterior (TA), and gastrocnemius (GC) of the lower limb on the stance-side while the subjects walked over the obstacle were selected. The activity levels of these muscles were examined.

The muscle activity signals from the individual muscles were processed into root mean square (RMS) values and quantified with reference to voluntary contraction (RVC) because RVC tends to decrease the sensitivity to differences in RMS EMG signals between individuals during moving activities such as gait. The average RVC values were taken from three repeated measurements, and the muscle activities during flat land walking in both types of shoes were determined as the RVCs of each experiment (Criswell, 2011). Thereafter, the experimental results were standardized as percentages of the measured $\mathrm{RVC}$ values (\%RVC).

\section{Task and procedure}

The subjects performed the experiments wearing high-heel shoes $(7 \mathrm{~cm})$ and flat shoes $(0 \mathrm{~cm})$. The constructed obstacles had adjustable heights. The obstacle heights were set to $10 \%, 20 \%$, and $30 \%$ of the length between the anterior superior iliac spine (ASIS) and the medial malleolus (MM) of each subject. For example, if the lower limb length was $80 \mathrm{~cm}$, the $10 \%, 20 \%$ and $30 \%$ obstacle heights were $8 \mathrm{~cm}, 16 \mathrm{~cm}$, and $24 \mathrm{~cm}$, respectively. The lower limb length was determined by measuring the length from the most prominent point in front of the pelvis in a supine position (ASIS) to the MM. The obstacles were $2 \mathrm{~m}$ long and $2 \mathrm{~cm}$ thick and were installed such that they could fall in the walking direction to prevent falls when a subject was caught by the obstacle.

The subjects performed 5 minutes of gait practice to acclimate to the laboratory and were instructed to walk at a normal pace. Subsequently, a starting point was marked. The subjects performed the gait practice repeatedly to ensure consistent gait habits. They were instructed to begin walking from the starting point when a signal was given and to move over the obstacle. Analysis began when subjects passed over the obstacle.

\section{Statistical analysis}

The experiments were conducted by randomly sampling the gait analyses results for each of the different shoes worn by the subjects and each of the different obstacle heights. The values were measured three times in each experiment, and the average value was used in analysis. Two-way repeated ANOVAs were used to compare the 
trunk and lower limb muscle activities while the subjects walked over obstacles of different heights between the high-heel shoe wearing and flat shoe wearing conditions. All statistical analyses were conducted using the SPSS version 21.0 program. P-values below .05 were taken to indicate significant differences.

\section{Results}

1. Comparison of muscle activity between high-heeled shoes and flat shoes depending on obstacle's heights

The results derived through the above mentioned study methods are shown in Table 1. Table 1 is a schematization of the \% RVC values of the stance-side trunk and lower limb muscle activities while walking over the obstacles of different heights were analyzed according to shoe type. In the present study, the differences in the muscle activities between the shoe types at the same obstacle height were not significant $(\mathrm{p}>.05)$.

2. Comparison of muscle activity according to obstacle's height in high-heeled shoes and flat shoes

In general, the supported side trunk and lower limb muscle activities increased as the obstacle heights increased regardless of shoe type. Considerable increases were identified in muscles other than the RA and the EO ( $p<05)$. Additionally, most of the analyzed data indicated greater

Table 1. Comparison of muscle activity between the types of shoes and among different obstacle heightswith high-heeled or flat shoes (\%RVC)

\begin{tabular}{|c|c|c|c|c|c|c|c|}
\hline \multirow{2}{*}{ Muscle } & \multirow{2}{*}{ Type of shoes } & \multicolumn{4}{|c|}{ Obstacle height } & \multirow{2}{*}{$\mathrm{F}$} & \multirow{2}{*}{$\mathrm{P}$} \\
\hline & & $0 \%$ & $10 \%$ & $20 \%$ & $30 \%$ & & \\
\hline \multirow{2}{*}{ RA } & Flat & $11.69 \pm 3.22$ & $12.27 \pm 2.87$ & $12.03 \pm 2.76$ & $12.30 \pm 2.95$ & .69 & .56 \\
\hline & High Heel & $12.03 \pm 2.72$ & $12.50 \pm 3.49$ & $13.11 \pm 3.10$ & $12.70 \pm 3.05$ & .69 & .56 \\
\hline \multirow{2}{*}{ ES } & Flat & $16.92 \pm 6.57$ & $20.66 \pm 9.24 *$ & $22.95 \pm 10.33^{\dagger+\S}$ & $24.03 \pm 10.10^{\ddagger \|}$ & 11.92 & .00 \\
\hline & High Heel & $19.91 \pm 6.16$ & $22.55 \pm 7.34^{*}$ & $23.89 \pm 8.73^{++\S}$ & $24.31 \pm 7.97^{\neq \|}$ & 11.92 & .00 \\
\hline \multirow{2}{*}{$\mathrm{EO}$} & Flat & $24.16 \pm 7.25$ & $25.75 \pm 8.51$ & $27.32 \pm 98.63^{\dagger+\S}$ & $26.50 \pm 8.30^{\neq}$ & 2.17 & .11 \\
\hline & High Heel & $29.27 \pm 8.65$ & $29.75 \pm 8.47$ & $31.51 \pm 9.37^{++\S}$ & $31.02 \pm 10.11^{\neq}$ & 2.17 & .11 \\
\hline \multirow{2}{*}{ IO } & Flat & $26.88 \pm 14.02$ & $29.41 \pm 13.75^{*}$ & $31.62 \pm 15.77^{+\dagger \S}$ & $31.93 \pm 14.42^{\neq \|}$ & 5.45 & .00 \\
\hline & High Heel & $28.06 \pm 9.93$ & $30.10 \pm 11.09^{*}$ & $32.85 \pm 13.97^{\dagger+\S}$ & $32.30 \pm 11367^{\neq \|}$ & 5.45 & .00 \\
\hline \multirow{2}{*}{$\mathrm{RF}$} & Flat & $18.50 \pm 7.02$ & $25.85 \pm 9.68 *$ & $27.02 \pm 9.58^{+}$ & $27.83 \pm 9.48^{\neq \| \uparrow}$ & 22.98 & .00 \\
\hline & High Heel & $25.74 \pm 9.38$ & $29.96 \pm 8.19^{*}$ & $32.05 \pm 8.67^{+}$ & $34.32 \pm 9.30^{\neq \| \uparrow}$ & 22.98 & .00 \\
\hline \multirow{2}{*}{ ST } & Flat & $26.70 \pm 7.79$ & $35.97 \pm 7.48^{*}$ & $37.59 \pm 7.79^{+}$ & $41.87 \pm 9.19^{\neq \| \uparrow}$ & 13.28 & .00 \\
\hline & High Heel & $30.92 \pm 9.50$ & $39.19 \pm 9.86^{*}$ & $40.56 \pm 10.29^{+}$ & $42.75 \pm 12.88^{\neq \| \uparrow}$ & 13.28 & .00 \\
\hline \multirow{2}{*}{ TA } & Flat & $59.05 \pm 16.47$ & $64.58 \pm 16.89^{*}$ & $68.41 \pm 16.42^{+}$ & $72.73 \pm 16.68^{\neq \| \uparrow}$ & 9.21 & .00 \\
\hline & High Heel & $55.13 \pm 18.07$ & $59.40 \pm 16.93^{*}$ & $56.70 \pm 15.76^{+}$ & $61.59 \pm 17.74^{\neq \| \uparrow}$ & 9.21 & .00 \\
\hline \multirow{2}{*}{ GC } & Flat & $70.28 \pm 29.45$ & $78.17 \pm 30.02 *$ & $86.48 \pm 36.37^{\dagger+\S}$ & $86.70 \pm 29.29^{\ddagger \|}$ & 11.85 & .00 \\
\hline & High Heel & $78.33 \pm 20.69$ & $89.84 \pm 28.13^{*}$ & $94.08 \pm 28.70^{++\S}$ & $102.61 \pm 29.85^{\neq \|}$ & 11.85 & .00 \\
\hline
\end{tabular}

* significant difference between $0 \%$ and $10 \%$ obstacle height, ${ }^{++}$significant difference between $0 \%$ and $20 \%$ obstacle height,

${ }^{\ddagger}$ significant difference between $0 \%$ and $30 \%$ obstacle height, $\S$ significant difference between $10 \%$ and $20 \%$ obstacle height,

"I "significant difference between $10 \%$ and $30 \%$ obstacle height, " significant difference between $20 \%$ and $30 \%$ obstacle height, Significance probability $\mathrm{P}<.05$. RA: Rectus abdominalis, ES: Erector spinalis, EO: External oblique abdominalis, IO: Internal oblique abdominalis, RF: Rectus femoris, ST: Semitendinosus, TA: Tibialis anterior, GC: Gastrocnemius 
muscle activity levels when high-heel shoes were worn. However, according to the \% RVC results for the tibialis anterior while the subjects walked over obstacles according to shoe type, greater muscle activities were observed when the subjects were wearing flat shoes than when they were wearing high-heel shoes.

\section{Discussion}

High-heel shoes induce changes in soft tissues around the feet by increasing the amount of vertical impact during standing and movement and may reduce muscle strength in the feet and ankle and damage the ligaments and joints. Because these changes may be detrimental to body arrangement and induce ankle instability, high-heel shoes are considered to be a cause of overall musculoskeletal system lesions (Garn and Newton, 1998). Additionally, high-heel shoes may greatly affect the mobility and stability of the feet, and such changes are also related to motor control abilities. Because obstacle gaits are movements that require highly harmonized joint movements in which the lower limbs should accurately contact the ground during the stance phase to maintain body balance, and the lower limbs should finely adjust the movements during the swing phase (Chesnin et al., 2000). Therefore, the eccentricconcentric contractions that continuous occur in the muscles around the ankle reflect the continuous muscle contraction activity and should be directly related to muscle fatigue (Svantesson et al., 1998). Gait is frequently affected by external environment factors during daily living activities. Therefore, based on previous studies, the diagnosis and evaluation of gait in special situations of daily living, such as the negotiation of environmental obstacles, is important (Fajen and Warren, 2003).

In the present study, the differences in muscle activity levels according to shoe type at the same obstacle height were not significant. However, most of the results of the comparison of the stance-side trunk and lower limb muscle activities while the subjects wore shoes with different heel heights indicated greater activities while wearing the high-heel shoes. Interestingly, according to the tibialis anterior \% RVC results, higher muscle activities were present when the subjects wore flat shoes than when they wore high-heel shoes. The calf muscle activity increased when the high-heel shoes were worn, while the activity of the tibialis anterior when flat shoes were worn was increased. These results indicate that the foot and ankle muscles were imbalanced and that the muscles were not contracting at the appropriate times, which reduced stability. This condition can cause musculoskeletal system problems throughout the entire body (Gefen et al., 2002; Yu et al., 2013). These differences in muscle activity are considered to be attributable to differences in kinematic phenomena caused by the relatively greater plantar flexion of the foot when walking in high-heel shoes compared with flat shoes. When woman are wearing a pair of high-heel shoes, the center of gravity of her upper body moves forward and the lower limb center of gravity moves backward (Snow and Williams, 1994). This supposition is supported by the results of the present study, which indicated that the activities of the stance-side trunk and lower limb muscles increased when the subjects walked over the obstacles. Specifically, the above result can be explained by the results that have indicated that the muscles continuously contract to maintain ankle joint stability (Malmir et al., 2017) and can be understood as resulting from the body's dynamic adaptation, which manifests as a state of plantar flexion while wearing high-heel shoes (Foster et al., 2012).

The present study has several limitations. First, because the present study evaluated muscle activities in young women, the results may not be generalized to people in all age groups. Second, the effects of high-heel shoe wearing were evaluated with a focus on activities of the stance-side trunk and lower limb muscles the while subjects 
walked over obstacles. Third, dynamic elements, such as changes in foot pressure and force moments, which may be imposed on the feet and the ankles and kinematic elements related to joint movements and changes in the movements were not measured. Therefore, the results of the present study may not be applied to all aspects of the feet and ankles. Therefore, studies measuring the effects of high-heel shoe wearing that incorporate a wider range of conditions and address these limitations should be performed.

\section{Conclusion}

The results of this study indicated that when subjects wore high-heel shoes, the stance-side trunk and lower limb muscle activities increased, and the activities of the muscles around the ankle increased, whereas the activity of the tibialis anterior decreased when the subjects walked over the obstacles. Therefore, based on the results of the present study, wearing high-heel shoes may easily cause trunk and lower limb muscle fatigue and may specifically weaken the tibialis anterior. In relation to these issues, we examined how human movements are affected by environmental factors to identify the effects of such movements on the musculoskeletal system and to provide basic data that are necessary for understanding the musculoskeletal system and providing useful information for future studies in related areas.

\section{References}

Abbud GA, Li KZ, DeMont RG. Attention requirements of walking according to the gait phase and onset of auditory stimuli. Gait Posture. 2009;30(2):227-32.

Blanchette MG, Brault JR, Powers CM. The influence of heel height on utilized coefficient of friction during walking. Gait Posture. 2011;34(1):107-10.

Chesnin KJ, Selby-Siverstein L, Besser MP. Comparison of an in-shoe pressure measurement device to a force plate: Concurrent validity of center of pressure measurement. Gait posture. 2000;12(2):128-33.

Cowan DN, Robinson JR, Jones BH, et al. Consistency of visual assessments of arch height among clinicians. Foot Ankle Int. 1994;15(4):213-7.

Criswell E. Cram's Introduction to Surface Electromyography (2nd ed). USA. Jones and Bartlett Publishers. 2011.

Edwards L, Dixon J, Kent JR, et al. Effect of shoe heel height on vastus medialis and vastus lateralis electromyographic activity during sit to stand. J Orthop Surg Res. 2008;3:2.

Fajen BR, Warren WH. Behavioral dynamics of steering, obstacle avoidance, and route selection. J Exp Psychol Hum Percept Perform. 2003;29(2):343-62.

Foster A, Blanchette AG, Chou YC, et al. The influence of heel height on frontal plane ankle biomechanics: implications for lateral ankle sprains. Foot Ankle Int. 2012;33(1):64-9.

Garn SN, Newton RA. Kinesthetic awareness in subjects with multiple ankle sprains. Phys Ther. 1998;68(11): 1667-71.

Gefen A, Meqido-Ravid M, Itzachak Y, et al. Analysis of muscular fatigue and foot stability during high-heeled gait. Gait posture. 2002;15(1):56-63.

Han JT. Comparison of Spatio-temporal Gait Parameters between Paretic and Non-paretic Limb while Stepping over the Different Obstacle's Heights in Subjects with Stroke. J Korean Soc Phys Med. 2014;9(1):69-74.

Lee MH, Chang JS, Lee SY, et al. The Effects of High-heeled Shoes on Static Balance and EMG Activity of Lower Extremity Muscles for Young Women. J Korean Soc Phys Med. 2009;4(1):43-8.

Malmir K, Olyaei GR, Talebian S, et al. Effects of Peroneal Muscles Fatigue on Dynamic Stability Following Lateral Hop Landing: Time to Stabilization vs. 
Dynamic Postural Stability Index. J Sport Rehabil. 2017;17:1-21.

Menz HB, Lord SR. Footwear and postural stability in older people. J Am Podiatr Med Assoc. 1999;89(7):346-57.

Powell J, Wilins D, Leiper J, et al. Stay on your feet safety walks group. Accid Anal Prev. 2000;32(3):389-90.

Power CM, Body LA, Torburn, L, et al. Stair ambulation in persons with transtibial amputation: An analysis of the Seattle Light Foot. J Rehabil Res Dev. 1997; 34(1):9-18.

Said CM, Goldie PA, Patla AE, et al. Effect of stroke on step characteristics of obstacle crossing. Arch Phys Med Rehabil. 2001;82(12):1712-9.
Snow RE, Williams KR. High heeled shoes: their effect on center of mass position, posture, three-dimensional kinematics, rear foot motion, and ground reaction forces. Arch Phys Med Rehabil. 1994;75(5):568-76.

Snow RE, Williams KR, Holmes GB. The effects of wearing high heeled shoes on pedal pressure in women. Foot Ankle. 1992;13(2):85-92.

Svantesson U, Osterberg U, Thomeé R, et al. Muscle fatigue in a standing heel rise test. Scand J Rehabil Med. 1998;30(2):67-72.

Yu J, Cheung JT, Wong DW, et al. Biomechanical simulation of high-heeled shoe donning and walking. J Biomech. 2013;46(12):2067-74. 
